\title{
Le corps dansant, trace d'un passé individuel et collectif, dans Tango de Carlos Saura
}

\author{
STÉPHANIE HONTANG
}

\begin{abstract}
Body and Dance: Traces of Individual and Collective Past in
Carlos Saura's Tango. It is difficult to ascertain at what moment precisely modern cinema was born: it is not easy because there are different kinds of modern movies. Here I want to talk about "modern cinema" and the spirit of the films made after the Second World War when directors tried to expose the annihilation and the horror of Auschwitz and Hiroshima. Started by the Italians with the "neorealismo" in the 1950s, this modern conception of making films is still present nowadays. But because the horrors of the $20^{\text {th }}$ century cannot be considered as a spectacle, we face two problems: what is the place of the moral in the film making process and where are the limits in the esthetics of representing horror. In Carlos Saura's Tango (1998), a musical film, we find these two questions and the way he answers them: the Spanish director has tried to represent the collective as well as the individual memories of the victims of Franco's and Videla's cruel dictatorships. His way? Music and body expression.
\end{abstract}

Keywords: Carlos Saura; Tango; dance; memory; intermediality; violence

On a souvent pu distinguer en cinéma la valeur esthétique de la valeur politique. Comme si l'une ne pouvait cohabiter avec l'autre au sein d'une œuvre pour défendre une même idée. Dès ses premiers pas en tant que réalisateur, l'espagnol Carlos Saura s'est ingénié à mener une réflexion aussi bien sur la forme que sur le fond au sein d'une filmographie engagée. A partir des années 80 , quelques années après la fin de la dictature franquiste, le cinéaste parvient à prolonger l'expérience dans ses films musicaux quand ceux-là sont généralement assimilés au genre du divertissement.

Au-delà d'une simple œuvre sur la genèse du tango argentin, le film musical Tango, réalisé en 1998, traite plus largement de la barbarie humaine et de sa représentation par un travail sur la mémoire historique aussi bien individuelle que collective. Carlos Saura exprime son traumatisme de la guerre civile lorsqu'il était encore enfant à travers le propre traumatisme de son double à l'écran, le protagoniste et metteur en scène Mario Suarez, dont la mémoire de la dictature de Videla est réactivée au moyen de numéros musicaux alliant tango 
et danse contemporaine. Le film de Saura s'inscrit totalement dans la veine et les objectifs du cinéma moderne né des horreurs de la seconde guerre mondiale. Aussi ce nouveau cinéma s'est-il donné comme défi celui de représenter la disparition et la violence. La violence n'étant pas un spectacle, cela pose le problème d'une morale de la forme au cinéma et les limites d'une esthétisation de l'horreur. Comment représenter ce que personne n'a vu ? Comment l'éthique s'associe-t-elle à l'éthique dans un travail nécessaire sur la mémoire historique ? La comédie musicale ou l'expression du corps dansant semble être la modalité choisie par Saura pour évoquer des sujets sensibles comme la dictature argentine, franquiste et la barbarie en général.

Pour mener à bien cette réflexion, nous allons nous appuyer tout particulièrement sur deux séquences clefs. Il s'agit de deux numéros de danse et musique tangueras. Ils interviennent dans la seconde partie du film. Ce sont les séquences $n^{\circ} 43$ et $n^{\circ} 45$. L'intérêt de ces représentations réside dans leur double nature. Nous assistons, d'une part, aux répétitions du spectacle final recréant des scènes de tortures. D'autre part, nous avons à faire aux rêveries du protagoniste Mario Suárez. Aussi la modalité musicale et corporelle choisie par Saura/Suárez va-telle évoluer pour que le spectateur puisse davantage mesurer toute sa puissance narrative et évocatrice.

Saura va choisir, dans un premier temps, un tango silencieux et désincarné pour nous parler de la violence. C'est l'impression qui se dégage de la séquence $\mathrm{n}^{\circ} 43$. Il n'y a pas d'introduction. La scène débute in medias res, comme si l'on suivait le flux de la pensée du personnage et metteur en scène Mario. C'est aussi une façon d'introduire cette violence, que la couleur rouge vive qui illumine le mur de fond et la surface de la scène relève avec force. La piste brille sous l'effet de la lumière à contre-jour, comme si du sang venait de couler. Ainsi, des silhouettes noires s'élancent dans ce bain de pourpre, tout en formant des couples. Les corps sont aplanis et se désagrègent sous l'effet des ombres chinoises. On n'entend que la musique des corps qui s'entrechoquent, des pas qui frappent au sol et des corps qui tombent sur la piste rouge, comme si l'on avait supprimé la voix, l'organe humain par antonomase. La séparation du geste de la voix semble sonner comme une amputation. Tout est synonyme de fracture dans cette scène. Par ailleurs, malgré des corps dessinés en ombre chinoise, on lit les mouvements et les pas de danse. Les figures de tango, synonyme de sensualité, revêtent un sens nouveau. Les pas, les mouvements et les enlacements sont agressifs. Les tours sont répétitifs et étourdissants, une impression que les panoramiques horizontales accentuent. En effet, la caméra balaie la scène de 
gauche à droite, puis de droite à gauche. Il s'agit d'un plan séquence. Aucune pause n'est tolérée. Les cavalières qui subissent les mouvements sont soumises à un rythme de danse soutenu. La répartition traditionnelle des rôles au sein du couple est exacerbée. Le partenaire ne domine plus mais torture sa cavalière. Cette dernière ne suit plus mais subit et souffre en silence. Les mains sur les corps des danseuses sont menaçantes. Le point d'appui des portées ne sont pas celles d'un tango ou d'une danse de couple. Les corps des danseuses sont précipités en l'air. Puis, ils retombent violemment sur les torses ou le haut des hanches de leurs partenaires. Certaines sont projetées au sol ou dans les bras d'un nouveau bourreau. Ce sont des postures peu confortables. Les figures que sont les renversées, ou « caídas », les « sentadas », les fentes arrières, les « enganches $\gg$ sont exagérées. En effet, les membres s'étirent ou se contorsionnent au maximum comme pour suggérer l'idée de corps écartelés ou fracturés. En somme, les danseurs exacerbent les figures de tango afin de signifier la torture, de créer des images de souffrance et de violence.

Si le désordre semble dominer au début, aussi bien au niveau du placement des danseurs que de la tenue des danseuses que les cavaliers tentent de maitriser, l'ordre s'installe progressivement. Le débit des pas ralentit dans les derniers temps de la phrase. Puis, les trois couples réalisent un canon : les danseurs reprennent et exécutent de manière décalée dans le temps un mouvement. Enfin, les deux couples restant dans le champ de la caméra réalisent la pose finale et clôturent la représentation. L'éclairage scénique, la progression du débit et du travail des corps au sein du numéro de danse viennent d'écrire un récit : l'instauration de la dictature après la répression. La danse en silence ainsi que la sobriété des plans bichromatiques rendent l'histoire fort saisissante, car seule la violence peut résonner au milieu de cet espace vide. Aucun élément ne vient parasiter ou adoucir la séquence. La sobriété dans l'utilisation des arts scéniques va de pair avec celles des moyens cinématographiques, car le plan séquence se construit sur la phrase, unité de base dans l'écriture de la chorégraphie (Robinson 1981 : 99). Le mouvement de la caméra épouse simplement les déplacements des danseurs.

A l'issue de la séquence, la caméra cadre le visage de Mario, alors allongé sur un lit au côté de sa nouvelle amante et danseuse Elena, un lit installé dans le studio de répétition. Il repense à la dictature militaire de Videla :

La imaginación te coloca barreras permanentemente y te impiden que llegues a la profundidad del horror. Sin embargo a mí me vienen cataratas de imágenes de amigos que ya no están. Fueron muchos miles de vidas, que se perdieron con esta represión brutal y descontrolada. Yo me fui muy joven de acá, relativamente joven. Me quedé muchos años en Europa, y cuando volví, la mayoría de mis 
Le corps dansant, trace d'un passé individuel et collectif, dans Tango de Carlos Saura

amigos habían desaparecido. [...] Lo que somos es lo que vivimos. (Saura 1998 : 1h50')

D'après le personnage, il semble qu'une représentation trop abstraite ou froide ne soit pas en mesure de rendre compte de la barbarie humaine. Le double de Saura va tenter de relever le défi en imaginant un nouveau numéro scénique plus réaliste comme pour mieux dominer les fantômes qui le hantent. C'est d'ailleurs sur cette image fantasmagorique que s'ouvrira la séquence $n^{\circ} 45$.

Toujours en proie à ses souvenirs, Mario, alors allongé dans le lit, tient fort Elena dans ses bras. L'image est filmée dans un plan d'ensemble. Le mur de fond translucide laisse apparaitre l'avancé progressive de silhouettes noires surdimensionnées vers un nouvel espace du studio. La panoramique horizontale allant de la gauche vers la droite suit la traversée de ces ombres, de derrière le mur translucide placé derrière le lit de Mario à une immense scène plongée dans une atmosphère crépusculaire. La paroi perpendiculaire au mur de fond fait figure de frontière transitoire entre l'espace de la chambre, symbolisant l'imagination de Mario, et la scène réaliste où va se dérouler un nouveau numéro de danse. En effet, les ombres se métamorphosent en hommes vêtus de treillis, comme si les spectres hantant l'esprit de Mario s'échappaient de son imagination pour s'incarner en êtres de chair et d'os. En redonnant vie à des fantômes, Saura/Suárez opère en quelque sorte un retour en arrière comme pour suggérer un vécu à l'origine de ces spectres. La transition entre les deux séquences fait figure de flashback. En effet, grâce à un mouvement de panoramique et la disposition en angle perpendiculaire de deux panneaux translucides créant des images spectrales, Carlos Saura recrée l'effet d'un fondu enchainé. Le réalisateur s'empare des arts scéniques pour traduire le langage cinématographique, quand dans un film plus classique on aurait eu recours à un fondu enchainé pour exprimer un retour en arrière ou l'entrée dans une séquence onirique. L'impression visuelle qui en découle est puissante, car on gagne en réalisme, en immédiateté au regard de procédés cinématographiques tel que le fondu enchainé qui est plus artificiel et indirect. La nature de la transition entre la séquence $n^{\circ} 44$ et la séquence $n^{\circ} 45$ signe le pacte de lecture avec ce prochain numéro. Les arts scéniques, dont la danse contemporaine puisant son inspiration dans le tango argentin, vont s'exprimer pleinement pour se mettre au service d'une évocation de la mémoire individuel de Mario quant à son expérience traumatisante de la dictature militaire. 
Le metteur en scène jette sur la scène non seulement ses spectres, sous la forme d'un groupe armé, mais aussi celle qui partage sa vie et ses blessures passées. C'est donc au milieu de personnages collectifs tels que les militaires qu'Elena va évoluer, dans un premier temps, au sein de la scène. En exposant sa moitié, Mario Suarez va exprimer en quelque sorte à travers elle sa subjectivité. C'est un « je » qui va s'opposer à un « nous » tout au long du numéro. Alors que les hommes habillés en treillis entament une danse militaire, Elena fait irruption dans le premier plan depuis le hors-champ, dans un regard-caméra, reléguant le groupe d'hommes au second plan dans une très faible profondeur de champ. Ainsi, le gros plan sur le visage de la danseuse relève avec force ses traits qui se contractent sous l'effet de la peur et de la douleur. L'échelle de plan choisie crée une grande proximité entre le spectateur et le personnage féminin. Cela peut provoquer un certain malaise, tant l'émotion est exacerbée et mise à nu de manière si proche de l'écran. Elena se met à pleurer mais la musique extradiégétique étouffe les gémissements. La caméra n'enregistre que l'image de la douleur et non le son. Le contraste créé entre le silence et l'« image affection» est puissant (Deleuze 1983 : 96). Toutefois, il établit une distance par rapport à l'action présente : une jeune fille terrifiée par un ballet militaire. Le personnage d'Elena va perdre en réalisme. L'immédiateté du bruit des pleurs a été supprimée au montage au profit d'un chœur appartenant à la bande son extradiégétique. Il s'agit de voix sans paroles qui dans un mouvement ascendant passent du grave à l'aigu. L'absence de textes, la hauteur du son et l'harmonie dissonante accompagnant le choeur produisent des voix fantomatiques. Grâce à une synchronisation entre image et musique, Elena est identifiée à ce chant déshumanisé. Par ailleurs, le personnage féminin va rester à l'écart du groupe de danseurs militaires. A l'issue du regard caméra, elle tourne le dos au spectateur, s'avance lentement vers le ballet placé au second plan. Puis, Elena s'arrête face à eux telle une spectatrice, comme s'il y avait eu une frontière imaginaire. Les deux entités restent indifférentes l'une par rapport à l'autre.

La danseuse les regarde. Toutefois, il n'y a pas de contacts physiques, ni d'échanges de regard. D'un point de vue chorégraphique, Carlos Saura crée une dissymétrie. Le ballet d'hommes exécute une marche militaire, introduite par un roulement de tambour. La marche du tango est donc remplacée par une marche martiale, que l'absence de syncopes souligne fort bien. Tous les temps sont marqués. Les danseurs évoluent en quinconce. Cette disposition permet de noter tous les gestes saccadés, mécaniques, et brefs. Il y a également un travail directionnel, que les mouvements de panoramiques et de travellings rendent compte. Les danseurs exploitent toutes les directions : horizontales, obliques et verticales, comme pour mieux insister sur le contrôle total de l'espace. La répétition et la symétrie des mouvements chorégraphiques du ballet militaire 
créent une armée de clones robotisés. A l'inverse, l'entrée d'Elena sur scène est marquée par l'explosion des sentiments de douleur et de peur dans « cette image-affection $\gg$. Le plan fixe, qui marque la durée, s'oppose à l'énergie inhumaine des militaires. Lorsqu'elle se dirige vers le groupe d'hommes, sa marche est lente et la trajectoire est aléatoire. C'est un personnage dominé par ses émotions. Elle incarne la subjectivité, l'individualité face une logique impersonnelle et collective. D'ailleurs, à l'issue de la première partie du numéro, Elena prend la fuite dans le côté droit du cadre de la caméra, comme pour tenter de fuir l'image de la danse militaire.

La course rapide filmée en travelling latéral reproduit la spontanéité, la peur et le refus de la part du personnage de se soumettre à la logique militaire. En effet, alors que la caméra donne à voir le tracé des pas des danseurs dominant l'espace dans toutes ses directions, on perd le sens de la vectorialité lors de la course à toute allure d'Elena filmée dans un plan taille. L'espace scénique apparaît alors comme immense. Elle traverse un champ de ruines. Un plan large donne à voir des murs de fond de grandes dimensions, de couleur marron foncé et opaques. L'espace est encombré de carcasse de voitures et de débris de grandes tailles. Paradoxalement, c'est un espace immense et reconstituant un lieu extérieur qui apparait comme fermé. Elena se retrouve comme « enfermée dehors $\gg$. Plus elle tentera d'évoluer sur l'immense scène, plus son espace vital et de circulation va se restreindre. A l'issue de la première partie du numéro, on constate que le réalisateur espagnol joue sur des contrastes. La dissociation entre l'image et le son, entre le personnage collectif et la solitude d'Elena, entre la froideur et l'émotion, entre un espace ouvert et fermé recouvrent différents sens. D'une part, le décalage entre le son et l'image souligne une distance temporelle. La scène n'évolue pas dans une perspective immanente mais transcendante avec ces personnages fantomatiques. La première partie de la représentation apparait davantage comme la projection d'images-souvenirs qui affleurent à la conscience de Mario. Il n'y a pas de logique entre les deux entités qui évoluent sur la scène comme si l'on suivait finalement le flux de la pensée du double de Saura. De plus, le personnage d'Elena réactive grâce son hypersensibilité la mémoire individuelle et le traumatisme de Mario. D'autre part, Carlos Saura exprime l'impossible expression de la subjectivité en mettant en scène une dualité où la violence militaire et la logique impersonnelle l'emportent face à l'individualité. Elena ne peut s'exprimer. Sa voix est étouffée. Elle n'a pas d'autre choix que de fuir. Seule l'image cinématographique révèle la censure.

Cette scène va se reproduire trois nouvelles fois au cours de la représentation. Tout d'abord, à l'issue de sa course, Elena se retrouve face à la formation d'une fosse commune par des militaires. La caméra la montre de dos, dans un plan pied. Puis, un coupage cut rompt avec le plan. Un travelling avant vient alors 
resserrer le cadre sur le visage d'Elena dans un très gros plan. Carlos Saura reproduit avec une légère différence le même plan analysé précédemment. Il exacerbe l'effet de proximité avec le personnage et l'extrême expressivité qui se dégage de ce visage en pleurs, dont on ne perçoit toujours pas les sons. Une plongée révèle ensuite l'horreur du charnier. Puis, lors de la dernière occurrence de ladite scène, le même mouvement de caméra resserre à nouveau le cadre sur le personnage dans un très gros plan. Suite à un raccord regard, la caméra subjective nous donne à voir la reconstitution d'un peloton d'exécution. Des danseurs sont disposés en ligne le long d'un mur de fond rouge. Les corps réalisent une tension, puis une détente musculaire, l'un après l'autre. Ils simulent ainsi la chute d'un corps après le coup du fusil. Ils le font de plus en réalisant un canon, ce qui reproduit l'image d'une exécution sommaire. Ainsi, le regard d'Elena constitue une fenêtre sur des tableaux de l'horreur. D'ailleurs la configuration des plans du charnier et du peloton d'exécution rappelle celle d'un cadre de peinture. Dans les deux cas, la disposition présente la même singularité : le cadre de la caméra ne cadre pas avec les bords de ces scènes indicibles. De plus, l'oeil de la caméra reste à distance et refuse tout travelling avant, alors que le procédé est employé sur le regard larmoyant d'Elena. La distance focale et le refus du recadrage par un décadrage semblent rappeler l'adage du réalisateur Jacques Rivette, qui dans son article « De l'abjection » pour la revue Les cahiers du cinéma en juin 1961 affirme que «le travelling est une affaire de morale » (Rivette 1961: 54, 55). De cette façon, Carlos Saura pose la question d'une morale de la forme au cinéma. Le réalisateur ne peut traiter ces plans comme les autres. On ne peut esthétiser l'horreur et le spectateur ne peut accepter de regarder cela comme un spectacle. C'est pourquoi, les gros plans sur le visage de la danseuse sont des regards caméras. C'est une manière d'interpeller le spectateur et de rompre avec l'illusion cinématographique afin de lui rappeler qu'il n'est pas face à un spectacle divertissant et banal, mais devant un film sur la mémoire et la dénonciation de la barbarie humaine. La forme choisie par Carlos Saura est peut-être une réponse à la réflexion de Mario qui affirmait que l'imagination ne permettait pas de prendre la mesure de l'horreur. Il semble qu'il y ait des limites à l'esthétisation de la violence. Par ailleurs, si l'on s'en tient au premier niveau de lecture, les intra-iconicités, que constitue l'articulation des gros plans sur Elena avec les tableaux de l'horreur, reproduisent par cet effet de répétition le traumatisme et les images obsessionnelles qui hantent les pensées de Mario.

Les « images-affections » ne seront pas les seules à porter la mémoire de Mario. Le corps porte aussi la trace d'une violence passée et va réactiver la mémoire en dansant. C'est la thèse du critique Mercè Saumell à propos du personnage d'Elena : 
Volviendo al tema de la corporalidad, Daniel Sibony analiza de forma brillante el papel del cuerpo coreográfico en su libro Le corps et la danse (1995) al sugerir los conceptos de cuerpo-memoria y cuerpo-evento. La película TANGO muestra fílmicamente este efecto a través de códigos teatrales: el recuerdo existe dentro de la memoria de Mario y la bailarina lo transmite, ella recrea el evento (las escenas de tortura y ejecuciones) a través de su cuerpo. (Saumell 2014: 3)

On peut étendre cette analyse à l'ensemble des danseurs sur scène qui vont reproduire également les scènes de tortures et d'exécution à travers leurs corps. Suite à la découverte du charnier, Elena prend à nouveau la fuite. C'est à partir de ce moment-là que la danseuse va entrer en contact physiquement avec les personnages collectifs. Une plongée donne à voir la traversée à contrecourant d'Elena au milieu d'une foule encadrée par des militaires. Les corps s'entrechoquent, et crée un effet de ralentissement. Puis, la scène de la séquence $\mathrm{n}^{\circ} 43$ en ombre chinoise se répète. Une fois après avoir traversé la marée humaine, Elena se retrouve face à un second obstacle. Trois hommes vêtus d'un treillis happent trois femmes de leurs mains et leurs imposent une danse violente. Les pas de tango sont à nouveau travestis en armes de tortures. Elena fait partie des trois danseuses. A la différence de la séquence $n^{\circ} 43$, on perçoit clairement les corps détendus des femmes tels des pantins qui se plient, volent en l'air et tombent sous l'effet des gestes brutaux de leurs cavaliers, qui tentent de les retenir dans leurs fuites. Ils ne dansent pas en harmonie. Le plan fixe permet d'apprécier les mouvements vifs et brusques. L'espace immense semble d'ores et déjà se réduire tel un entonnoir. Tout d'abord, la vue du peloton d'exécution annule toute perspective dans le champ de la caméra et scelle le destin d'Elena. La danseuse est emmenée en dernier lieu dans un espace intérieur.

Aucune échappatoire ne semble possible à présent pour le personnage féminin. Le lieu s'apparente à une salle d'interrogatoires et de tortures. En effet, la dernière partie de la représentation s'ouvre sur l'image d'une chaise posée au centre de la pièce et éclairée par la très faible lumière d'une lampe de plafond. Lors de l'entrée en scène de la première victime et de ses bourreaux, l'éclairage s'intensifie donnant à voir un espace cloisonné de toutes parts. Seul le mur de fond laisse entrevoir deux entrées de chaque côté, deux espaces intermédiaires entre le couloir de la mort et la salle de torture. Un corps à corps s'engage alors entre les bourreaux et les victimes qui défilent sur la scène et la chaise de torture. Les « golpes » ne marquent pas une entrée sur scène mais terrorisent les prisonniers allongés au sol. Les «enganches » ne sont plus synonyme de sensualité mais servent à tordre les corps des danseuses que l'on installe violemment sur la chaise. Les prisonniers relâchent musculairement. Ils ne résistent plus à l'apesanteur et tombent au sol. Cela ressemble visuellement à 
une petite mort, l'horizontalité du corps s'opposant à la verticalité, signe de vie et d'énergie. Il y a un jeu également entre lâché/prise. Le bourreau empoigne et jette sa victime. Il la torture et la pousse au sol. Les corps des danseurs se tordent, se tendent et se relâchent pour mieux exprimer une douleur. En somme, on note non seulement un travail sur le poids mais aussi sur les lignes du corps, afin de montrer des corps abimés, torturés et qui perdent la vie sous l'effet de la torture. Il est intéressant de remarquer comment la caméra suit la logique de l'écriture chorégraphique qui est structurée par «le contraste », « la diminution», « la répétition $\gg$, et le travail de l'espace en trois dimensions (Robinson 1981 : 79, 97). En effet, la séquence met tout d'abord en scène une dualité entre Elena et des personnages collectifs, une dualité qui se transforme en une danse contact lors de la scène de l'interrogatoire. Quant à la caméra, elle offre des plans larges pour mettre en avant les jeux de contraste : la course d'Elena à contrecourant, des danses en désaccord...Puis, le cadre va se resserrer, une évolution parallèle à celle des corps qui se touchent pour s'entrechoquer ensuite dans un corps à corps. Par ailleurs, les travellings et les panotravellings permettent d'apprécier l'espace dans toutes ses directions. Les mouvements suivent ainsi de près les déplacements subis ou voulus par les danseurs. La caméra danse et souffre avec les personnages. En somme, les moyens cinématographiques viennent redoubler l'effet de la chorégraphie, comme pour mieux frapper l'esprit du spectateur. Toutefois, à l'issue du numéro, la caméra va à nouveau se centrer sur un seul personnage.

Une fois l'interrogatoire terminée, Elena se détache du corps de ballet, alors qu'elle s'était fondue progressivement jusqu'à présent dans la masse de victimes. La danseuse se précipite au fond de la scène. L'image est floue au premier plan, quand la profondeur de champ est nette à l'arrière-plan, ce qui met en valeur le visage apeurée d'Elena, comme si l'on tentait de dissocier les deux plans. En réalité, la danseuse reprend le rôle de spectatrice qu'elle avait endossée au début de la séquence, signant le retour à une certaine subjectivité. Le personnage va sortir de son isolement du fond de la scène, tant le spectacle de l'horreur lui est insupportable. Cependant, au lieu de fuir, elle court vers les militaires comme pour les implorer de mettre fin à la violence. Ses déplacements sur scène sont filmés en caméra libre, reproduisant ainsi la précipitation vers le groupe d'hommes. De plus, l'effet de regard loupe semble reproduire la vue d'Elena obstruée par ses larmes et qui cherche son chemin au milieu de la scène. Ainsi, la caméra nous met à la place de ce personnage et nous transmet à nouveau ses émotions. A l'issue de sa course, la jeune femme se jette dans les bras d'un bourreau. Mais ce geste fraternel échoue, l'homme restant impassible. Le glas résonne. Puis, à l'instar de ses compagnons, le corps vertical et encore plein de vie d'Elena se détend et s'écroule au sol. Carlos Saura traduit dans cette 
scène fort expressive tout son pessimisme face à l'homme. Si l'on accepte l'identification du réalisateur au personnage du metteur en scène Mario, on peut comprendre cette séquence comme une projection de son traumatisme de la guerre civile alors qu'il était encore enfant. En effet, Mario projette son propre souvenir à travers une figure féminine en pleurs et au visage juvénile. La fragilité du personnage inhérent à son identité de femme et la naïveté de l'acte final fait d'Elena un personnage infantile. Ainsi, à travers la mémoire du régime militaire en Argentine, Carlos Saura réactive sa mémoire personnelle de la guerre civile espagnole et de la dictature franquiste. De plus, lorsqu'il confie au critique Marcel Oms ses « souvenirs de la guerre civile », il fait des va-et-vient entre les images traumatisantes de la foule terrorisée à l'annonce de la guerre, des rues de Madrid «devenues vides et silencieuses », du « silence oppressé », du «sifflement d'une bombe », de la « terreur cosmique », de la « sensation de peur devant l'inconnu », d'une « jolie fille blonde allongée sur le sol, les cheveux tachés de sang et le visage tranché par des éclats de vitre $\gg$ et les films où il a tenté de traduire ces images (Oms 1981 : 100-102). Il cite par exemple son œuvre Prima Angélica où il a voulu reproduire son souvenir de l'affolement qu'a suscité l'annonce de la guerre civile parmi la population madrilène (Oms 1981 : 100-102). Carlos Saura confie même vouloir réaliser un film sur la période allant de la guerre civile au début de la transition démocratique :

Un tel film contiendrait inévitablement beaucoup d'autobiographie et c'est la principale raison pour laquelle depuis quelques années j’ai récapitulé mes souvenirs accumulant des matériaux dispersés qui puissent me servir un jour de base à la rédaction du scénario. Les extraits [...] n'ont autres unités de correspondre aux trois étapes de mon périple, de notre périple familial, pendant les années de la guerre et correspondent à Madrid, Cheste (Valence), et Barcelone. (Oms $1981: 100-102)$

Ces confessions datent de 1992. Elles confirment que l'œuvre de Carlos Saura est hantée par ses souvenirs traumatisants de la lutte intestine, au point de devenir un projet de film. Au regard de cela, on peut avancer d'une part la légitimité de voir dans les images de la séquence précédente montrant des personnages torturés, souffrants, effrayés, et affolés une dimension autobiographique. Tango a été réalisé en 1998. Ainsi, l'œuvre peut constituer une partie du projet saurien, bien qu'elle se matérialise à travers la dictature de Videla.

Néanmoins, au-delà de la dimension personnelle, cette dernière scène de la séquence revêt une dimension plus générale puisqu'elle constitue une évocation universelle de la barbarie et de la guerre tant au niveau musical que visuel. Dès 
l'introduction, on entend un roulement de tambour qui est omniprésent au cours de la représentation. Il rythme la marche martiale et la scène du peloton d'exécution. Lors de l'entrée des danseurs dans la salle de torture, un cor résonne et rappelle les sonneries militaires. Le motif joué par l'instrument est repris par des cuivres plus timbrés. Les sons sont plus doux. Il s'agit du trombone et de la trompette qui évoquent la foule, la multitude. Le glas ferme la séquence musicale et de danse, pour signifier la mort du personnage d'Elena. Il y a une mise en scène de l'émotion chez l'auditeur grâce à un travail musical qui s'associe à celui de la danse contact et des moyens cinématographiques. Le message sonore est clair et déstabilisant. Les connotations militaires et funéraires facilitent la lecture sonore. De plus, le choix d'une composition polytonale et dissonante ne peut que traduire un malaise chez l'auditeur. En effet, tous les motifs se croisent et ne permettent pas d'identifier une mélodie. Cela permet d'évoquer le désordre et le tourment auxquels sont soumises les victimes de toute violence. A l'instar de la réflexion menée sur la genèse tanguera et les stéréotypes qui l'entourent, Carlos Saura a recours à nouveau aux clichés musicaux dans la séquence $n^{\circ} 45$ afin de transmettre un message clair. En réalité, la séquence musicale dans son ensemble constitue une évocation universelle de la barbarie et de la guerre. La dimension universelle réside également dans l'aspect visuel, voire pictural et chorégraphique. Les cris sourds d'Elena enfermée dans un espace sombre, cloisonné de toutes parts, et éclairé par la seule lumière de lampes à la forme conique rappellent la configuration et les personnages du tableau de Guernica peint par Pablo Picasso. C'est un élément de plus dans la thèse d'un film sur les souvenirs traumatisants de la guerre civile par le Carlos Saura enfant. L'intericonicité établie avec le tableau du peintre espagnol est aussi une façon de donner une dimension universelle au film Tango, la peinture ayant été reprise maintes fois pour dénoncer la barbarie humaine dans le monde. Par ailleurs, le tango argentin laisse place à la danse contemporaine dans la séquence. Le choix d'une mise en scène théâtralisée de l'espace de danse dans la dernière partie de la représentation inscrit la séquence dans la veine du Tanztheater de la chorégraphe allemande Pina Bausch, dont l'objectif était de faire passer du sens à travers un mouvement pour atteindre la plus grande expressivité et intensité. Carlos Saura semble mettre à profit les enseignements de l'artiste allemande pour élaborer un numéro fort expressif afin que le spectateur prenne la mesure de l'horreur. La référence à Pina Bausch s'exprime même en termes d'intericonicité. Les plans montrant les danseurs en train de se poignarder ou la vue sur le charnier sont des citations de son spectacle Le sacre du printemps, que la chorégraphe a imaginé à partir du travail du compositeur et chef d'orchestre russe Stravinsky. En élaborant un dialogue inter-arts et interculturel, Carlos Saura abaisse les frontières 
Le corps dansant, trace d'un passé individuel et collectif, dans Tango de Carlos Saura

géographiques comme pour « réconcilier dans un même élan révolutionnaire tous les peuples opprimés » (Pageaux 2002 : 78) et dessiner « les contours d'un nouvel internationalisme terrestre $\gg$ (ibid.) autour de l'élaboration et de la représentation d'une mémoire collective des victimes de la barbarie humaine dans le monde.

A l'instar des films de Roberto Rossellini, Carlos Saura relève là l'un des enjeux que s'est donné le cinéma moderne au lendemain de la seconde guerre mondiale. Ainsi, le réalisateur espagnol reconstitue non seulement l'histoire du cinéma dans la forme mais aussi dans le fond. Il va même plus loin en la complétant grâce à la danse contemporaine et la relation qu'il établit entre la photo, la peinture et le cinéma. La danse accompagnée de la musique participe d'un art cinématographique qui se veut toujours plus expressif et libre. Elles viennent en dernier lieu définir le septième art comme une discipline de l'image en mouvement qui tend vers la tridimensionnalité. Toutefois, le cinéaste n'en oublie pas de rappeler les limites de cette verve artistique. Pour Saura, le cinéma doit être aussi militant, ce qui pose la question de la représentation. L'artiste doit faire preuve d'éthique tout comme le spectateur. C'est pourquoi, le réalisateur a fait des choix formels très variés au sein de son film s'adaptant ainsi à chaque débat : la genèse tanguera, l'Autre et le genre, la mémoire personnelle et collective. Si l'on s'en tient à la relation qu'établit Carlos Saura entre les deux pays hispaniques, l'Argentine apparait pour lui comme un miroir de la guerre civile espagnole qui l'a tant marquée, ainsi que de ses propres formations personnelles et artistiques. Ainsi, le film Tango articule la double dimension autobiographique et universelle, et continue à faire de l'Argentine un espace de migrations notamment dans le domaine artistique comme si la mixité, inhérent à la genèse tanguera, faisait partie de toute identité.

\author{
Stéphanie Hontang \\ stephanie.hontang@sfr.fr \\ Université de Pau et des Pays de l'Adour \\ Avenue de l'Université BP 576 \\ $64012 \mathrm{Pau}$ \\ FRANCE
}


HONTANG

\section{Bibliographie}

Deleuze, G. 1983. L'image mouvement. Paris : Editions de minuit.

Jullier, L. 2012. Analyser un film, de l'émotion à l'interprétation. Paris : Flammarion.

Oms, M. 1981. Carlos Saura. Paris : Ed. Edilig.

Pageaux, D.-H. 2002. Eléments pour une géosymbolique. Littérature Générale et Comparée et Géographie. - J. Bessière, Savoirs et littérature. Literature, The humanities and the social sciences. Paris : Presse Sorbonne nouvelle, 75-92.

Rivette, J. 1961. De l'abjection. - Les cahiers du cinéma, 120, 54-55.

Robinson, J. 1981. Eléments du langage chorégraphique. Paris : Vigot.

Sanchez Vidal, A. 1993. Retrato de Carlos Saura. Barcelona: Galaxia Gutenberg.

Sanchez Vidal, A. 1998. El cine de Carlos Saura. Madrid: Caja de Ahorros de la Inmaculada de Aragón.

Saumell, M. 2014. Teatralidad, metateatralidad y dramaturgia en el cine de Carlos Saura, http://lanimal.org/image/memoria/139/pdf/080101-139-14.pdf (21.12.2014).

Saura, C. 2005. Tango. SND.M6.DVD. 1h50'.

Siron, J. 2002. Dictionnaires des mots de la musique. Paris : Autre mesure.

Thibaudeau, P. 2011. Le couple, l'intime et le politique : quelques exemples dans le cinéma de Carlos Saura. - Actes du Colloque International L'intime et le politique dans la littérature et les arts contemporains. Paris : Michel Houdiard Editeur, 225-235. 